\title{
Key Role of Rotated Domains in Oxygen Intercalation at Graphene on $\mathrm{Ni}(111)$
}

\author{
Luca Bignardi $^{1}$, Paolo Lacovig ${ }^{1}$, Matteo M. Dalmiglio ${ }^{1}$, \\ Fabrizio Orlando ${ }^{1} \ddagger$, Aliakbar Ghafari ${ }^{1} \S$, Luca Petaccia $^{1}$, \\ Alessandro Baraldi ${ }^{1,2,3}$, Rosanna Larciprete ${ }^{4}$, Silvano Lizzit ${ }^{1}$ \\ ${ }^{1}$ Elettra - Sincrotrone Trieste, s.s. 14, km 163.5, 34149 Basovizza, Trieste, Italy. \\ ${ }^{2}$ Università degli Studi di Trieste, via Valerio 2, 34127 Trieste, Italy \\ ${ }^{3}$ Istituto Officina dei Materiali - CNR, s.s. 14, km 163.5, 34149 Basovizza, Trieste, \\ Italy \\ ${ }^{4}$ Istituto dei Sistemi Complessi - CNR, via dei Taurini 19, 00185 Roma, Italy \\ E-mail: silvano.lizzit@elettra.eu
}

7 April 2017

\begin{abstract}
Herein, we provide a complete description of the intercalation of oxygen at the strongly interacting graphene on $\mathrm{Ni}(111)$, highlighting the role of rotated graphene domains in triggering the intercalation. High-resolution core-level photoelectron spectroscopy provided a full characterization of the interface at each stage of the intercalation, revealing the formation of an oxide layer between graphene and the metal substrate. Angle-resolved photoemission spectroscopy measurements showed that the oxide decouples efficiently graphene from the substrate, restoring the Dirac cone and providing a slight $\mathrm{n}$-doping. Photoelectron diffraction experiments revealed that graphene domains not aligned with the Ni substrate are the first to be intercalated with oxygen and are preferential regions under which the oxygen is retained during the deintercalation.
\end{abstract}

PACS numbers: 73.22.Pr, 81.05.ue, 73.20.-r, 73.20.At, 79.60.-i, 79.60.Jv, 68.43.Fg, 68.65.Pq

Keywords: Graphene, Intercalation, Photoelectron Spectroscopy, Graphene-Metal Interfaces.

Submitted to: 2D Mater.

$\ddagger$ Present address: Paul Scherrer Institut, 5232 Villigen PSI, Switzerland

$\S$ Present address: Helmholtz-Zentrum Berlin fr Materialien und Energie GmbH, Hahn-MeitnerPlatz 1, 14109 Berlin, Germany 


\section{Introduction}

The understanding of the nature of the graphene-metal interaction and the possibility of tuning the electronic properties of graphene (GR) on metals are considered a crucial step towards the inclusion of graphene into devices, paving the way towards a graphenebased electronics on a large industrial scale [1-4]. Despite the broad interest raised in the past ten years, this topic still faces both applicative challenges and unresolved fundamental issues.

Chemical Vapour Deposition (CVD) is currently the most exploited protocol to epitaxially grow graphene on transition metals, on account of the high quality of the final product and of the simplicity and reproducibility of the process, making it a suitable candidate for a standardized graphene growth protocol [1,5-7]. On the other hand, the presence of a metallic substrate is causing strong modifications in graphene's electronic properties, which can differ greatly from those of a self-standing layer.

The intercalation of atomic species between the graphene and the metal has been proven as a successful way to modify the metal-adsorbate interaction, significantly leading to the engineering of the graphene band structure and giving the possibility to regain the features of free-standing graphene by accurate choice of the intercalant. Furthermore, the intercalation can ease the possibility of transferring graphene flakes to other substrates without the employment of aggressive chemicals, which could damage the graphene and alter its peculiar features [8-18].

The intercalation of oxygen has proven to be successful for graphene-passivated metal surfaces upon a suitable choice of $\mathrm{O}_{2}$ partial pressure and substrate temperature, regardless the extent of the graphene-metal interaction. As a remarkable example, weakly interacting graphene on $\operatorname{Ir}(111)$ was successfully intercalated with oxygen, which decoupled the GR from the substrate and lifted the mini-gaps in the $\pi$ band due to a moiré-induced periodic potential [19-21]. Likewise, the reversible intercalation of oxygen under graphene grown on the more strongly-interacting $\mathrm{Ru}(0001)$ surface has been achieved, restoring its nearly free-standing character [22-25].

Epitaxial graphene on $\mathrm{Ni}(111)$ has been attracting wide interests, in reason of a remarkable spin-filtering capability of this interface and of a possible employment in spin-based devices, which could take advantage of the large spin relaxation length in graphene [26-32]. However, the intercalation of oxygen at GR/Ni(111) was believed to be very challenging to obtain, since graphene has nearly the same lattice parameter of the underlying substrate and the interaction with $\mathrm{Ni}$ is very strong, owing to the hybridisation of the $\mathrm{Ni} 3 d$ states with the $\pi$ band of graphene [9]. In view of this, graphene was revealed to successfully protect and passivate the Ni surface and other metal layers intercalated between it and the Ni substrate [33,34]. Nevertheless, a recent publication has theoretically suggested the feasibility of the oxygen intercalation, drawing the reaction pathways for the $\mathrm{O}_{2}$ intercalation mechanism and showing how the graphene domain edges are of paramount importance for the penetration of oxygen under graphene [35]. Complementary to that, very recent experimental reports suggest 
the feasibility of the oxygen intercalation at GR/Ni(111), which leads to modifications in the features of intercalated graphene with respect to the pristine interface $[36,37]$.

In this work, we prove the feasibility of the intercalation of oxygen under a complete layer of epitaxial graphene on $\mathrm{Ni}(111)$, providing the conditions of $\mathrm{O}_{2}$ pressure and sample temperature to achieve it. We show that oxygen intercalation constitutes an effective way to decouple graphene from $\mathrm{Ni}(111)$ and to quench the strong substrateadsorbate interaction with the formation of a thin $\mathrm{Ni}$ oxide layer at the interface. Moreover, we highlight the role of the graphene domains not aligned with the substrate, which are the main feedthroughs to intercalate the oxygen at this interface [38-40].

The modifications to the interface during the entire process were investigated by means of high-resolution x-ray photoelectron spectroscopy (XPS), x-ray photoelectron diffraction (XPD) and angle-resolved photoemission spectroscopy (ARPES). We monitored in situ the evolution of the $\mathrm{C}$ 1s and $\mathrm{O}$ 1s core levels after the exposure to a flux of molecular oxygen in order to asses the change of their binding energy (BE) and shape for the oxidized interface. These outcomes were complemented by ARPES measurements revealing the formation of a self-standing graphene layer, decoupled from its metallic substrate. The stability of the intercalated interface was assessed by annealing the sample in vacuum, in order to evaluate any effects on the structure and electronic properties of the self-standing graphene. XPD provided a complete overview of the modifications of the structure features of the interface caused by the intercalation process.

\section{Experimental Methods}

High-resolution core-level spectra and x-ray photoelectron diffraction patterns were acquired at the SuperESCA beamline at the Elettra Synchrotron (Trieste, Italy) [41]. The experimental chamber is equipped with a SPECS Phoibos hemispherical electron energy analyzer (150 mm mean radius), integrated with an in-house developed delay-line detector. The base pressure in the UHV chamber was better than $10^{-10}$ mbar.

Valence band photoemission spectra were acquired at the BaDElPh beamline of Elettra [42] using a SPECS Phoibos 150 hemispherical analyzer combined with a 2D detector consisting of a phosphor screen coupled with a CCD commercial camera. The ARPES maps were measured with photon energy equal to $34 \mathrm{eV}$ in a $\pm 13^{\circ}$ wide angular region centred at the $\mathrm{K}$-point of the reciprocal space.

Samples were prepared in situ in each UHV chamber employed for the experiments. A $\mathrm{Ru}(0001)$ single crystal was cleaned in an ultra-high vacuum chamber (UHV) by several cycles of $\mathrm{Ar}^{+}$sputtering at $2.5 \mathrm{kV}$, followed by flash annealing up to $1600 \mathrm{~K}$. Nickel was evaporated from an electron bombardment evaporator on the Ru surface at $300 \mathrm{~K}$, followed by annealing up to $700 \mathrm{~K}$ to reorder the film surface. The Ni thickness, estimated by means of a quartz microbalance, was about 20 layers, corresponding to ca. $40.6 \mathrm{~nm}$. A clear $(1 \times 1)$ low-energy electron diffraction (LEED) pattern was observed after the Ni film deposition. No XPS signal originating from the Ru substrate 
was observed during all the experiments. Graphene was grown on $\mathrm{Ni}$ by thermal decomposition of ethylene $\left(P_{E t h}=1 \times 10^{-7} \mathrm{mbar}\right)$, while keeping the substrate at $900 \mathrm{~K}$, dosing ca. 70 Langmuir $\left(1 \mathrm{~L}=10^{-6}\right.$ torr $\left.\cdot \mathrm{s}\right)$.

Oxygen intercalation $\left(\mathrm{O}_{2}\right.$ purity: $\left.99.9991 \%\right)$ was achieved in a step-wise process by means of a gas doser, whose nozzle was placed in the vicinity of the sample surface $(<1 \mathrm{~mm})$, while keeping the sample at $470 \mathrm{~K}$. The oxygen back pressure in the experimental chamber was ca. $5 \times 10^{-4}$ mbar during the intercalation. The pressure at the sample surface was estimated to be an order of magnitude larger than the background pressure in the chamber. $\mathrm{C} 1 s$ and $\mathrm{O}$ 1s spectra were acquired after each step to track the progress of the intercalation. Three steps were necessary to achieve a saturation condition with no further modifications to the $\mathrm{C}$ 1s and $\mathrm{O}$ 1s spectra. The experimental data were fitted with a Doniach-Šunjić function [43] convoluted with a Gaussian distribution and a Shirley background. The binding energy scale is referred to the Fermi level measured in the same experimental conditions.

The XPD measurements have been performed at a photon energy of $400 \mathrm{eV}$. Each XPD pattern was produced by measuring 610 spectra in the $\mathrm{C} 1 \mathrm{~s}$ core level region over a wide azimuthal $(\phi)$ sector $\left(130^{\circ}\right)$ for polar angles $(\theta)$ ranging from grazing emission $\left(\theta=60^{\circ}\right)$ to normal emission $\left(\theta=0^{\circ}\right)$. For each of these spectra the peak fit analysis was performed and the intensity of each component resulting from the fit, i.e. the area under the photoemission line, was extracted. The resulting XPD patterns consist in the stereographic projection of the modulation function $\chi$, which is defined as

$$
\chi=\frac{I(\theta, \phi)-I_{0}(\theta)}{I_{0}(\theta)} .
$$

$I_{0}$ is the average intensity for each azimuthal scan at fixed polar angle.

The simulations were performed with the Electron Diffraction in Atomic Cluster (EDAC) package [44] for a free-standing, flat graphene layer.

The calculation of the reliability $(R)$ factor provided a quantitative comparison between simulated and experimental x-ray photoelectron diffraction patterns. The $\mathrm{R}$-factor is defined as [45]:

$$
R=\frac{\sum_{i}\left(\chi_{\exp , i}-\chi_{\mathrm{th}, i}\right)^{2}}{\sum_{i}\left(\chi_{\exp , i}^{2}+\chi_{\mathrm{th}, i}^{2}\right)},
$$

where $\chi_{\exp , i}$ and $\chi_{\mathrm{th}, i}$ correspond to the experimental and theoretical modulation functions, respectively. The sum index $i$ runs over all available data point at different angles. The lower the $R$ factor, the better the agreement between experiment and theory.

\section{Results and discussion}

Figure 1 displays high-resolution XPS spectra of the $\mathrm{C}$ 1s and $\mathrm{O}$ 1s core levels at different stages of the intercalation process. The spectra at the bottom were acquired on the pristine GR/Ni(111) interface. The main component resulting from the peak fitting of 


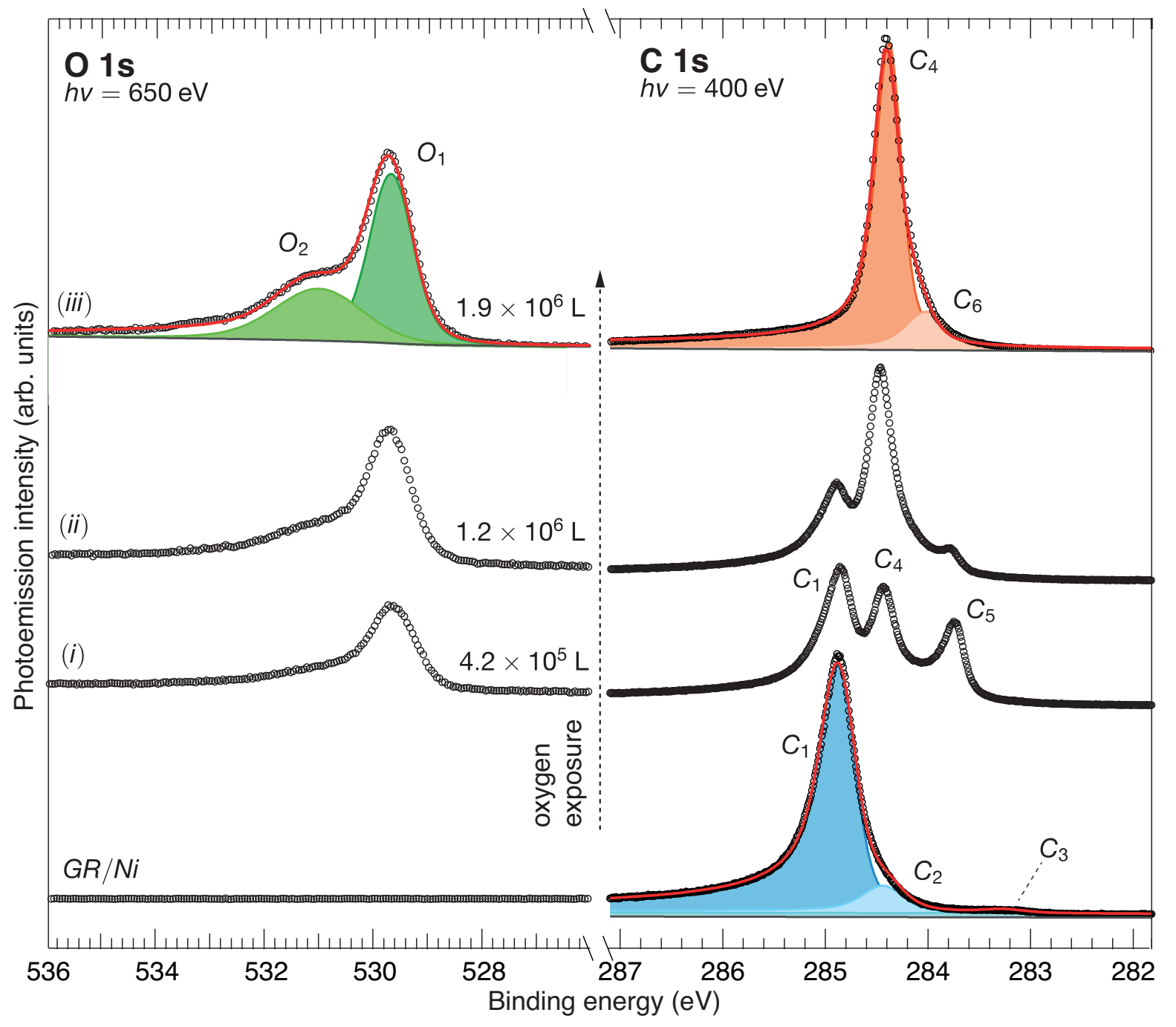

Figure 1. High-resolution $\mathrm{C}$ 1s and $\mathrm{O}$ 1s core level spectra acquired on graphene on $\mathrm{Ni}(111)$ and along the exposure to oxygen. The spectral contributions resulting from peak-fit analysis and the corresponding fit to the data (red line) are reported for the pristine sample and for the final stage (iii). For each step the total oxygen exposure (in Langmuir) is indicated. For an explanation of the peaks labelling refer to the text.

the $\mathrm{C}$ 1s level $\left(C_{1}\right)$ is centred at a binding energy $(\mathrm{BE})$ of $284.85 \mathrm{eV}$ and associated to graphene in direct contact with the $\mathrm{Ni}$ substrate $[9,39,46]$. This peak may include the contribution from domains both aligned and rotated with respect to the substrate orientation [39]. A less intense shoulder $\left(C_{2}\right)$ located at $284.45 \mathrm{eV}$ has been attributed to a fraction of rotated domains that are grown on regions where surface Ni carbides are present [39], while the small component $C_{3}$ at $283.2 \mathrm{eV}$ was assigned to surface $\mathrm{Ni}$ carbides.

A step-wise exposure to molecular oxygen while keeping the sample at $470 \mathrm{~K}$ remarkably affected the position and shape of the relevant core level spectra. Already at step (i) a significant amount of oxygen was detected by measuring the $\mathrm{O} 1$ s level, clearly 
showing the presence of a component $\left(O_{1}\right)$ at a $\mathrm{BE}$ of $529.7 \mathrm{eV}$, sided by a shoulder $\left(O_{2}\right)$ at higher binding energy $(531.15 \mathrm{eV})$. The two components were found to increase with oxygen exposure. The component $O_{1}$ is compatible both with chemisorbed oxygen on $\mathrm{Ni}(111)$ and with the formation of $\mathrm{NiO}$ [47-53]. The component $\mathrm{O}_{2}$ can be attributed either to the presence of regions of non-stoichiometric $\mathrm{NiO}$ rich in $\mathrm{Ni}^{3+}$ groups [47-52] or to oxygen belonging to the top layer of $\mathrm{NiO}[49,54]$.

Likewise, with exposure to oxygen two new components, $C_{4}(\mathrm{BE}=284.45 \mathrm{eV})$ and $C_{5}(\mathrm{BE}=283.79 \mathrm{eV})$, appeared along with the $C_{1}$ peak in the $\mathrm{C} 1$ s spectrum. The identical $\mathrm{BE}$ measured for $C_{2}$ and $C_{4}$ is a first indication that the latter component could originate from graphene with a weakened interaction with the substrate [39]. A longer exposure (ii) reduced the spectral weight of the components $C_{1}$ and $C_{5}$, while $C_{4}$ was greatly enhanced. After a further stage (iii), only the components $C_{4}$ and $C_{6}(\mathrm{BE}=284.05 \mathrm{eV})$ were observed. Remarkably, the full width at half maximum (FWHM) of the component $C_{4}(270 \mathrm{meV})$ appeared to be considerably smaller than the value observed for the main component $C_{1}$ on the bare nickel, which amounted to $420 \mathrm{meV}$. The FWHM and the shape of the $C_{4}$ peak are found to be comparable to the situation observed for GR/Ir(111), where the FWHM for C 1s measured in similar conditions was $250 \mathrm{meV}$ [55]. Furthermore, the BE of the component $C_{4}$ was found to have a value close to what observed for quasi free-standing graphene, only with a shift towards high BE of ca. $150 \mathrm{meV}$ [56].

Gathering these outcomes, the effects resulting from exposing GR/Ni(111) to molecular oxygen can be explained as follows. Molecular oxygen dosed in UHV on clean $\mathrm{Ni}(111)$ at the same sample temperature that we used for intercalation is known to undergo a dissociative chemisorption forming a $(2 \times 2)$ structure for coverages up to $0.25 \mathrm{ML}$. An intermediate chemisorbed phase may appear between 0.25 and 0.35 ML, with the formation of a $(\sqrt{3} \times \sqrt{3})$-R30 superstructure, while a higher coverage triggers the nucleation of $\mathrm{NiO}$ islands surrounded by surface areas with chemisorbed oxygen. The lateral growth and eventually the coalescence of these islands lead to the formation of a complete $\mathrm{NiO}$ layer, whose thickness has been estimated to be between 2 and 4 layers $[50-53,57]$.

The simultaneous presence of $O_{1}$ and $O_{2}$ in the $\mathrm{O}$ 1s spectrum indicates the presence of both chemisorbed oxygen on $\mathrm{Ni}$ and the formation of a $\mathrm{NiO}$ layer already at stage (i); therefore, it is reasonable to assign the $C_{4}$ component in $\mathrm{C} 1 \mathrm{~s}$ to graphene supported by an oxide layer. On the other hand, the component $C_{5}$, which is shifted by ca. $700 \mathrm{meV}$ towards lower $\mathrm{BE}$ with respect to $C_{4}$ can be attributed to graphene lying on regions where oxygen is still chemisorbed on the Ni surface.

When intercalating oxygen at the GR/Ir(111) interface, oxygen is only chemisorbed on the metal substrate, without forming an oxide layer. This results in a strong p-doping of graphene that was detected by ARPES (ca. $670 \mathrm{meV}$ ), consequently shifting the $\mathrm{C}$ 1s peak of a similar amount towards lower BE $[15,19]$. Hence, it is reasonable to assume that, for the oxygen intercalated $\mathrm{GR} / \mathrm{Ni}(111)$ at stage (i), the low BE position of peak $C_{5}$ owes to a similar p-doping effect arising from oxygen chemisorbed on the Ni surface. 

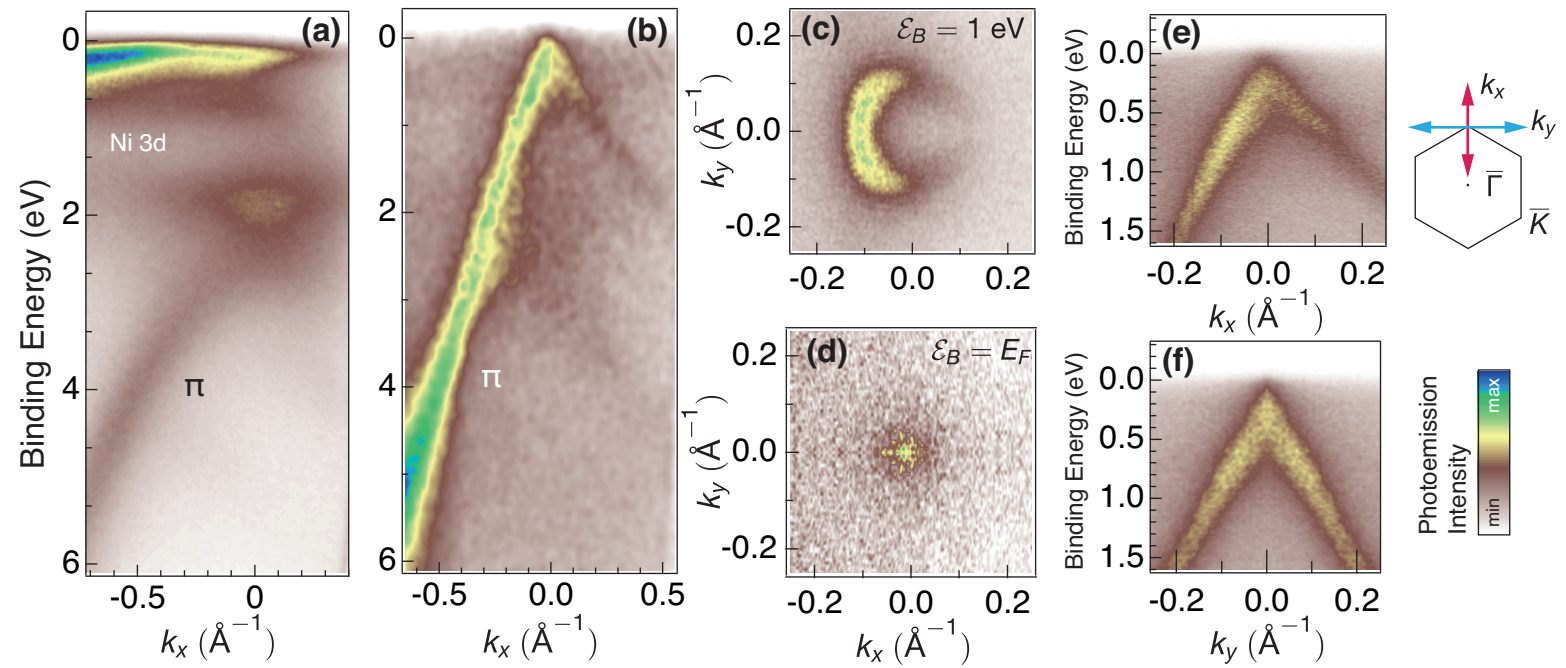

Figure 2. ARPES measurements of the band structure of pristine and lifted graphene on $\mathrm{Ni}(111)(h \nu=34 \mathrm{eV})$. The zero of the bottom axis corresponds to the $\bar{K}$ point of the GR Brillouin zone. (a) Band structure of GR/Ni around the GR $\bar{K}$ point of the graphene Brillouin zone (BZ) acquired along the $\overline{\Gamma K}$. (b) Band structure acquired around $\bar{K}$ along $\overline{\Gamma K}$ after oxygen intercalation. (c) and (d) Constant energy cuts at $E_{F}$ and $\mathcal{E}_{B}=1 \mathrm{eV}$ of the band structure of intercalated $\mathrm{GR} / \mathrm{Ni}$ around the $\bar{K}$ point. (e) and (f) Detail of the Dirac cone along $\overline{\Gamma K}$ and the direction ortogonal $\overline{\Gamma K}$, respectively. The relevant directions in the Brillouin zone are marked and displayed in the diagram at top right.

This is in agreement with a theoretical prediction indicating that a low coverage of oxygen chemisorbed on Ni significantly p-dopes the graphene layer [58].

The trend observed for peak series (ii) and (iii) validates this interpretation and is consistent with the mechanism of growth of $\mathrm{NiO}$ on $\mathrm{Ni}(111)$ [51]. As the oxygen dose increases, the $\mathrm{O}$ 1s signal shows the increase of the $\mathrm{NiO}$ peak. At the same time, the spectral weight of the $C_{1}$ and $C_{5}$ components is transferred to $C_{4}$ and $C_{6}$. This behavior could be accounted for by the lateral growth of the $\mathrm{NiO}$ islands at the expenses of the surface areas covered by chemisorbed oxygen. Graphene is then fully intercalated and lifted by the presence of the oxide, as the position and shape of $C_{4}$ indicate. The presence of $C_{6}$ can suggest that part of the graphene lies on regions where the $\mathrm{NiO}$ layer is nonhomogeneous or non-stoichiometric, producing a different doping than what observed for the majority of the graphene layer $\left(C_{4}\right)$. A quantitative analysis showed that during the intercalation the total area of the $\mathrm{C}$ 1s peak remains unaltered within the experimental error, then hinting at the complete lifting without etching of the graphene layer.

The effect of oxygen intercalation on the band structure of the interface was revealed by ARPES, which provided the spectral function of the system and strengthen the interpretation of the XPS spectra. ARPES measurements on pristine GR/Ni(111) acquired in the vicinity of the $\bar{K}$ point of the Brillouin zone of graphene along the $\overline{\Gamma K}$ direction are reported in Figure 2-(a). We identify the features of the Ni $3 \mathrm{~d}$ bands in the vicinity of $E_{F}$. Moreover, the graphene $\pi$ band is shifted towards high binding 
energy by ca. $2.5 \mathrm{eV}$ with respect to the position of free-standing graphene, with the Dirac cone dissolved into the metal $3 \mathrm{~d}$ band, because of the strong interaction and hybridisation between the $\mathrm{Ni} 3 \mathrm{~d}$ band and the graphene $\pi$ band in the region where they overlap [9].

After the last oxygen exposure (stage iii) the band structure was drastically modified, as depicted in Figure 2-(b). The hybridization with the $\mathrm{Ni} 3 \mathrm{~d}$ band was removed and the characteristic shape of the Dirac cone was restored and recognisable, having the Dirac point located near the Fermi level. This feature signals the strongly reduced interaction between graphene and the substrate with the formation of a nearly self-standing graphene layer [8, 19,59,60].

Panels (c) and (d) of Figure 2 show constant energy $\mathcal{E}_{B}$ cuts at $\mathrm{BE}=1 \mathrm{eV}$ and at $\mathrm{BE}=E_{F}$ (Fermi surface), respectively. The former displays the characteristic shape of the $\pi$ band for self-standing graphene [61]. The latter shows that the Fermi surface at $E_{F}$ is basically point-like, a further validation of a fully lifted interaction with the substrate and a restored quasi-free standing character of the graphene layer. This outcome is in agreement with recent electron energy loss measurements on oxygen intercalated GR/Ni(111) where the spectra displayed signatures of a freestanding graphene [36].

The spectral function was acquired in detail close to the Dirac point along $\overline{\Gamma K}$ and along the direction perpendicular to $\overline{\Gamma K}$ (Figure 2-e and 2-f, respectively), in order to directly inspect the shape of the Dirac cone. The dispersion of the Dirac cone and the position of the Dirac point were obtained by fitting the momentum distribution curves (MDCs) acquired in direction perpendicular to $\overline{\Gamma K}$ with two lorenzian profiles between $\mathcal{E}_{B}=0.7 \mathrm{eV}$ and $\mathcal{E}_{B}=E_{F}$ and subsequently by interpolating their maxima with a linear fit. A slight $n$-doping of graphene (ca. $120 \pm 30 \mathrm{meV}$ ) was observed. The extracted value for the Fermi velocity $v_{F}$ was found to be $1.10 \pm 0.15 \times 10^{6} \mathrm{~m} / \mathrm{s}$. We attribute the detected doping to the non uniform nature of the intercalated oxide layer. As mentioned earlier the formation of a non-uniform, non-stoichiometric oxide layer containing both $\mathrm{Ni}^{2+}$ and $\mathrm{Ni}^{3+}$ groups could lead to an inhomogeneous doping of the carbon layer. This feature, combined with the presence of defects and domain boundaries, might be responsible as well for the momentum broadening of the Dirac cone $\left(0.075 \AA^{-1}\right.$, measured at $\mathcal{E}_{B}=1 \mathrm{eV}$ along the direction perpendicular to $\overline{\Gamma K}$ ) when compared for example to GR/Ni(111) intercalated with $\mathrm{Au}$ [8]. Furthermore, it is worth noting that the difference between the position of the $C_{4}$ component and the $\mathrm{C}$ 1s for quasi-freestanding graphene [56] amounts to the doping level extracted from ARPES measurements, similarly to what observed for the weakly-interacting GR/Ir(111) [15].

The oxygen intercalated sample was annealead up to $680 \mathrm{~K}$ with a linear ramp of $0.5 \mathrm{~K} / \mathrm{s}$, in order to evaluate the stability of the oxide layer and to investigate the reversibility of the intercalation. The evolution of the $\mathrm{C}$ 1s peak was monitored during the annealing, as reported in Figure 3-(a). The $\mathrm{C}$ 1s core level of the lifted graphene remains unaltered up to ca. $500 \mathrm{~K}$. The trends observed for the different components of $\mathrm{C}$ 1s as reported in Figure 3-(b) allow the identification of different stages during the deintercalation process. The significant increase of $C_{6}$ at the expenses of $C_{4}$ reveals 

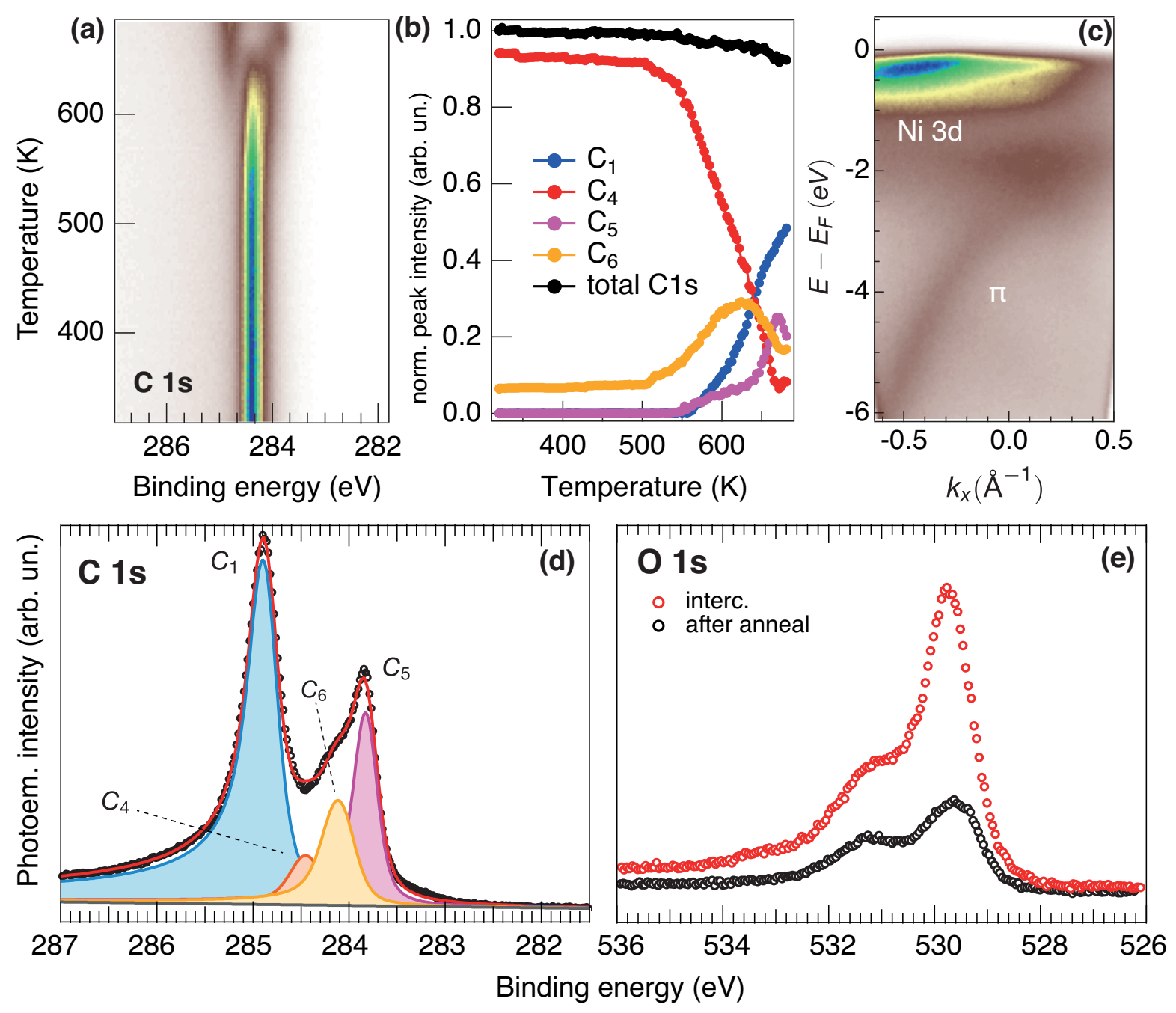

Figure 3. (a) Evolution of the $\mathrm{C} 1 \mathrm{~s}$ core level of the intercalated graphene vs. temperature during a vacuum annealing up to $680 \mathrm{~K}$. Inset: Photoemission intensity colours scale. (b) Evolution of the (normalised) total area of the components of the $\mathrm{C}$ 1s level during the annealing. (c) ARPES measurement on the deintercalated sample in the vicinity of the graphene $\bar{K}$ point. (d) High-resolution C 1s level acquired after the annealing. (e) $\mathrm{O} 1 \mathrm{~s}$ level acquired before and after the annealing.

that above $500 \mathrm{~K}$ the intercalated $\mathrm{NiO}$ layer undergoes some structural modifications due to the oxygen diffusion and formation of $\mathrm{Ni}_{2} \mathrm{O}_{3}$ groups $[47,52]$. When $T>550 \mathrm{~K}$, the increase of $C_{5}$ indicates the re-appearance of $\mathrm{Ni}$ regions on which the oxygen is chemisorbed, strongly p-doping the overlying graphene. The reappearance of the $C_{1}$ component at ca. $570 \mathrm{~K}$ reveals that patches of surface oxide undergo reduction, leaving clean metal surface areas on which graphene is landing back.

For $T>650 \mathrm{~K}$ further desorption of oxygen from the sample leaves more and more clean Ni regions on which the graphene is landed back, accounting for the raise of the $C_{1}$ component. However, the restoration of the $C_{1}$ intensity after annealing to $680 \mathrm{~K}$ is only partial because oxygen has not been completely desorbed at this temperature. 

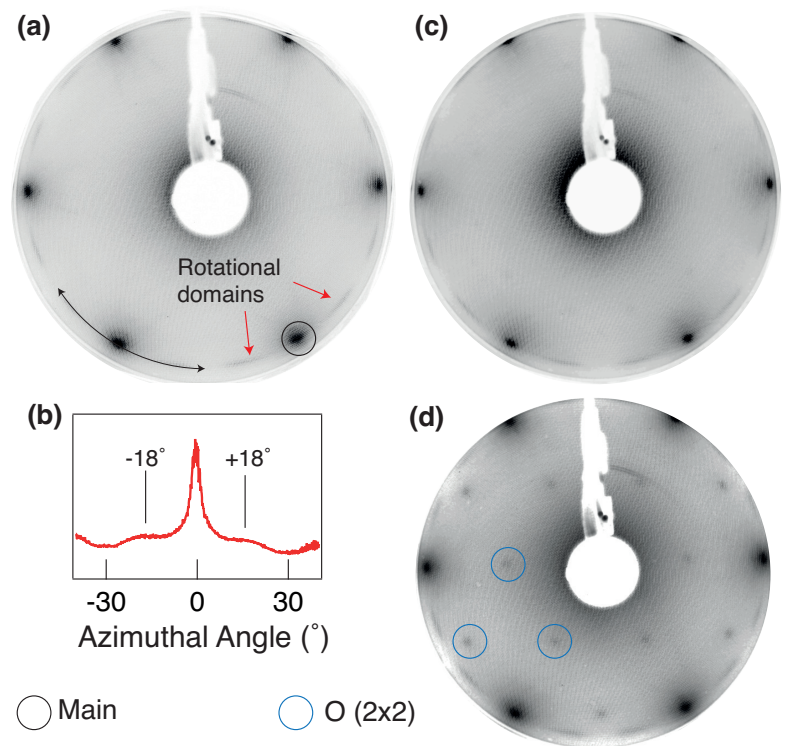

Figure 4. LEED pattern acquired (a) on pristine GR/Ni(111); (c) on oxygen intercalated $\mathrm{GR} / \mathrm{Ni}(111)$; (d) on the annealed sample. The energy of the primary beam was $72 \mathrm{eV}$. (b) Line profile taken along the black line from pattern (a).

Surprisingly, the total amount of carbon, as deduced from the C 1s total area, was reduced only by ca. $7 \%$. This proves that the graphene layer is only mildly etched when oxygen desorbs from the surface, this etching taking place above $550 \mathrm{~K}$ [62], as seen in Figure 3-(b). Such observation is sided by a reduction in the core-level $\mathrm{O} 1 \mathrm{~s}$ spectrum of the $\mathrm{O}_{1}$ component, associated to $\mathrm{NiO}$ groups, with respect to $\mathrm{O}_{2}$, as shown in Figure 3-(e), hinting then at a conversion of $\mathrm{Ni}^{2+}$ groups into $\mathrm{Ni}^{3+}$ groups [50-52] A quantitative analysis reveals that the total intensity of the $\mathrm{O}$ 1s level is reduced by more than $60 \%$ with respect to its original value.

ARPES measurements (Figure 3-c) carried out on the sample after annealing showed that the Dirac cone moves back well below $E_{F}$ regaining the situation observed for pristine graphene on $\mathrm{Ni}(111)$. Therefore, the contribution of the $C_{4}$ component in the ARPES measurements is no longer visible due to its low intensity, nor that from $C_{5}$, despite its sizeable intensity.

A further insight into the origin of the different $\mathrm{C}$ 1s components was given by the low energy electron diffraction (LEED) measurements, reported in Figure 4.

The pattern obtained for pristine GR/Ni is shown in Figure 4-(a). The expected $(1 \times 1)$ pattern typical of graphene on $\mathrm{Ni}(111)$ (framed with black circles) coexists with streaky features disposed symmetrical to the main LEED spots, (indicated by red arrows). Such features can be assigned to the presence of a small amount of rotated graphene domains in the overlayer, as shown by earlier literature [38,39]. By acquiring a line profile as shown in Figure 4-(b) we could observe that this distribution of rotated domains is centred at an azimuthal angle of $\pm 18^{\circ}$ with respect to the main orientation. 

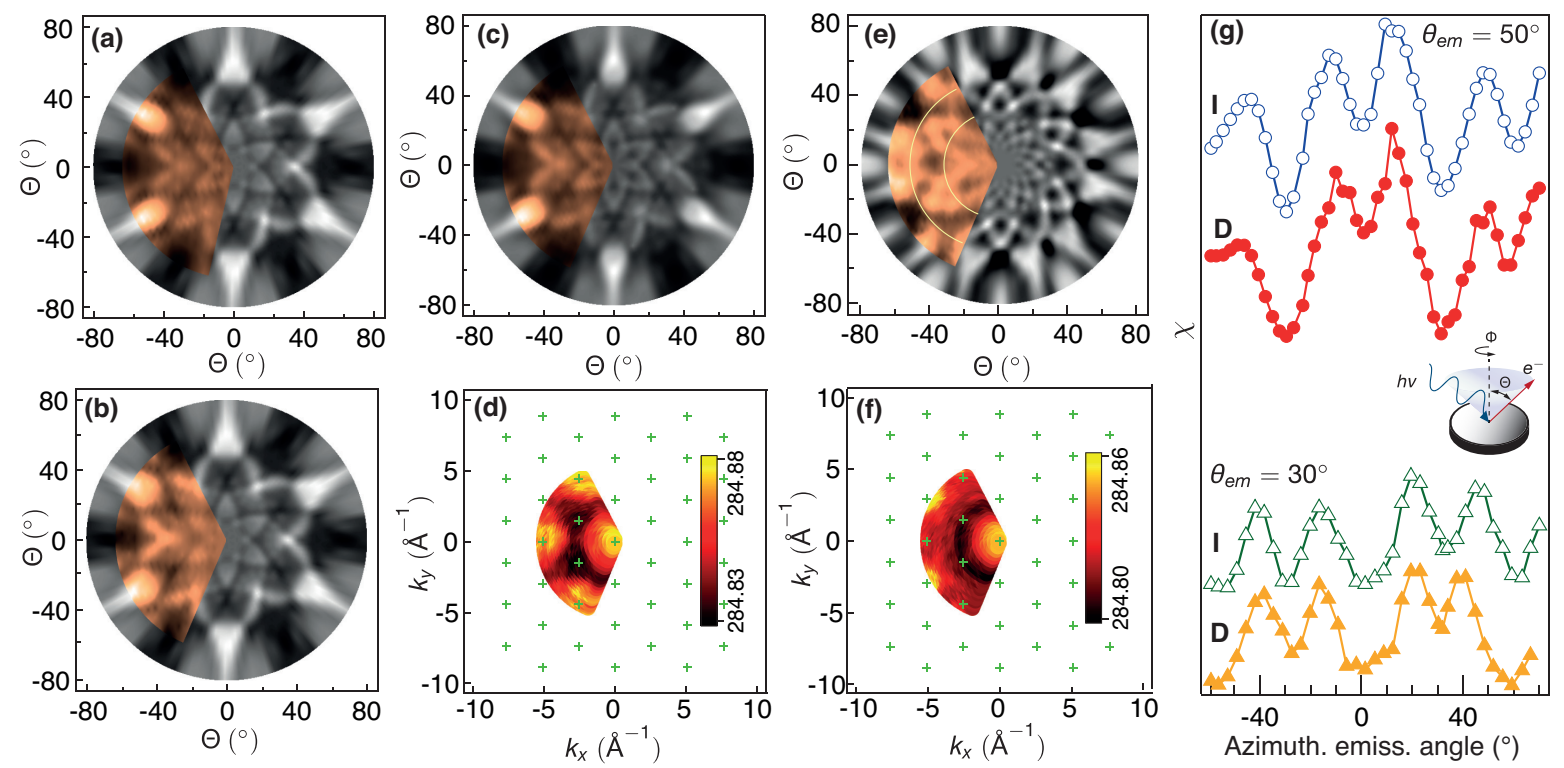

Azimuth. emiss. angle $\left({ }^{\circ}\right)$

Figure 5. Stereographic projection of the $\mathrm{C}$ 1s modulation function $\chi=\left(I-I_{0}\right) / I_{0}$ (see methods for details) as a function of emission angle and corresponding multiple scattering electron diffraction simulations for: (a) graphene on $\mathrm{Ni}(111)$; (b) intercalated GR/O/Ni(111); (c) and (e) peak $C_{1}$ and $C_{5}$ of the annealed sample, respectively. The continous lines in (e) indicate the azimuthal scans plotted for deintercalated sample in panel $(\mathrm{g})$. The coloured fraction of the disc is the data; the greyscale part is the multiple scattering calculation. Lighter color indicates higher intensity. (d) and (f) Binding energy modulations in reciprocal space observed for the peaks $C_{1}$ and $C_{5}$ of the deintercalated sample, respectively. The green crosses correspond to the reciprocal lattice $\Gamma$ points of graphene for component $C_{1}$. (g) Modulation function $\chi$ of the component $C_{5}$ acquired for azimuthal scans at $\theta_{e m}=50^{\circ}$ and $30^{\circ}$, at stage (i) of intercalation (I), as in Figure 1, and after the deintercalation (D), as in Figure 3-d. The schematic depicts the geometry of the XPD experiments.

Oxygen intercalation did not lead to appreciable changes to the LEED pattern Figure 4-(c). After sample annealing to deintercalate the oxygen, Figure 4-(d), the spots of a $(2 \times 2)$ overlayer were observed, framed with blue circles. This is in very good agreement with our interpretation of the XPS spectra, where the $\mathrm{C}$ 1s component at low binding energy $\left(C_{5}, \mathrm{BE}=283.79 \mathrm{eV}\right)$ is assigned to graphene intercalated with chemisorbed oxygen, which, as remarked previously, assumes on $\mathrm{Ni}(111)$ a $(2 \times 2)$ configuration.

A complementary overview of the structural properties of the interface came by acquiring XPD patterns, as displayed in Figure 5. Each pattern was assembled from the $\mathrm{C}$ 1s core level spectra collected over a wide emission angle sector (see Experimental Methods for details), for electrons emitted with a kinetic energy of ca. $115 \mathrm{eV}$. The geometry of the experiment is sketched in panel (g) of the same Figure. To determine the structure of the interface at each step of intercalation and deintercalation the experimental patterns (in color scale) were compared with multiple scattering photoelectron diffraction simulations (black and white scale) of a self-standing 
graphene layer, i.e. without considering any contributions from the substrate [44]. The assessment of the agreement between experimental and simulated pattern is obtained by the minimisation of the Reliability $(\mathrm{R})$ factor [45], as explained in the methods section.

Panel (a) in Figure 5 reports diffraction patterns measured for pristine $\mathrm{GR} / \mathrm{Ni}$, as stemming from the $C_{1}$ component. Similarly, panel (b) reports the pattern sourcing from the $C_{4}$ component observed for the intercalated sample. Panel (c) and (e) report the pattern associated with components $C_{1}$ and $C_{5}$ of the deintercalated sample, following the labelling already used in Figure 3. Last, in Figure 5-(g) we show the azimuthal scans of the component $C_{5}$ acquired at stage (i) of the intercalation and after deintercalation, at two different emission polar angles.

Remarkably, while the patterns (a-c) show features that can be immediately ascribed to graphene with a single orientation [55], the pattern shown in (e) differs considerably from what one could expect from a singly oriented graphene domain, indicating that it originates from a layer with unalike structural features. Such observation is confirmed by plotting in the reciprocal space the BE modulation of the components $C_{1}$ and $C_{5}$ for the deintercalated sample. These modulations correspond to the variations of the $\mathrm{BE}$ of the $\mathrm{C} 1 \mathrm{~s}$ core level components as function of the electron emission angles $(\theta, \phi)$ due to the core level band dispersion in graphene [55]. The $C_{1}$ component, Figure 5-(d), shows a modulation identical to what observed for singlyoriented graphene [55]. On the other hand, we attribute the modulation of peak $C_{5}$, Figure 5-(f), to graphene domains which are azimuthally rotated with respect to the layer associated with the component $C_{1}$. Recent publications $[38,39,63]$ have reported about samples of CVD-grown graphene on $\mathrm{Ni}(111)$ displaying a small fraction of rotated domains. We then assumed that such rotated domains could produce the pattern displayed in Figure 5-(d), by including in the corresponding simulated pattern contributions sourcing both from graphene aligned with the substrate $(\phi=0)$ and from domains oriented with an azimuthal offset $\pm \phi_{0}$. The total intensity of the simulated diffraction patterns can be written as:

$$
I_{\mathrm{tot}}=a I_{\text {align }}+b\left[\frac{1}{2} I_{\mathrm{rot}}\left(\phi_{0}\right)+\frac{1}{2} I_{\mathrm{rot}}\left(-\phi_{0}\right)\right] \quad(b=1-a)
$$

with $a, b$ representing the fraction of aligned and rotated domains, respectively, assuming that the $+\phi_{0}$ and $-\phi_{0}$ orientations are equally probable for symmetry reasons.

We then performed simulations with different values of $\phi_{0}$ and determined the contribution of rotated domains by calculating the value of the R-factor vs the $a$ and $b$ coefficients (Figure 6). For the diffraction pattern in Figure 5-(e), the minimum R-factor of 0.38 was found for a value of $\phi_{0}$ of $18^{\circ}$, which is in excellent agreement with our LEED measurements and comparable to what observed in earlier literature $[38,39,63]$. In this pattern the rotated domains are dominating (75\%) over the aligned domains, as shown in Figure 6-(d). The different azimuthal orientation of the graphene domains associated with the component $C_{5}$ is thus the reason for which no contributions belonging to these domains can be seen in the ARPES spectrum shown in Figure 3-(c). Moreover, the 


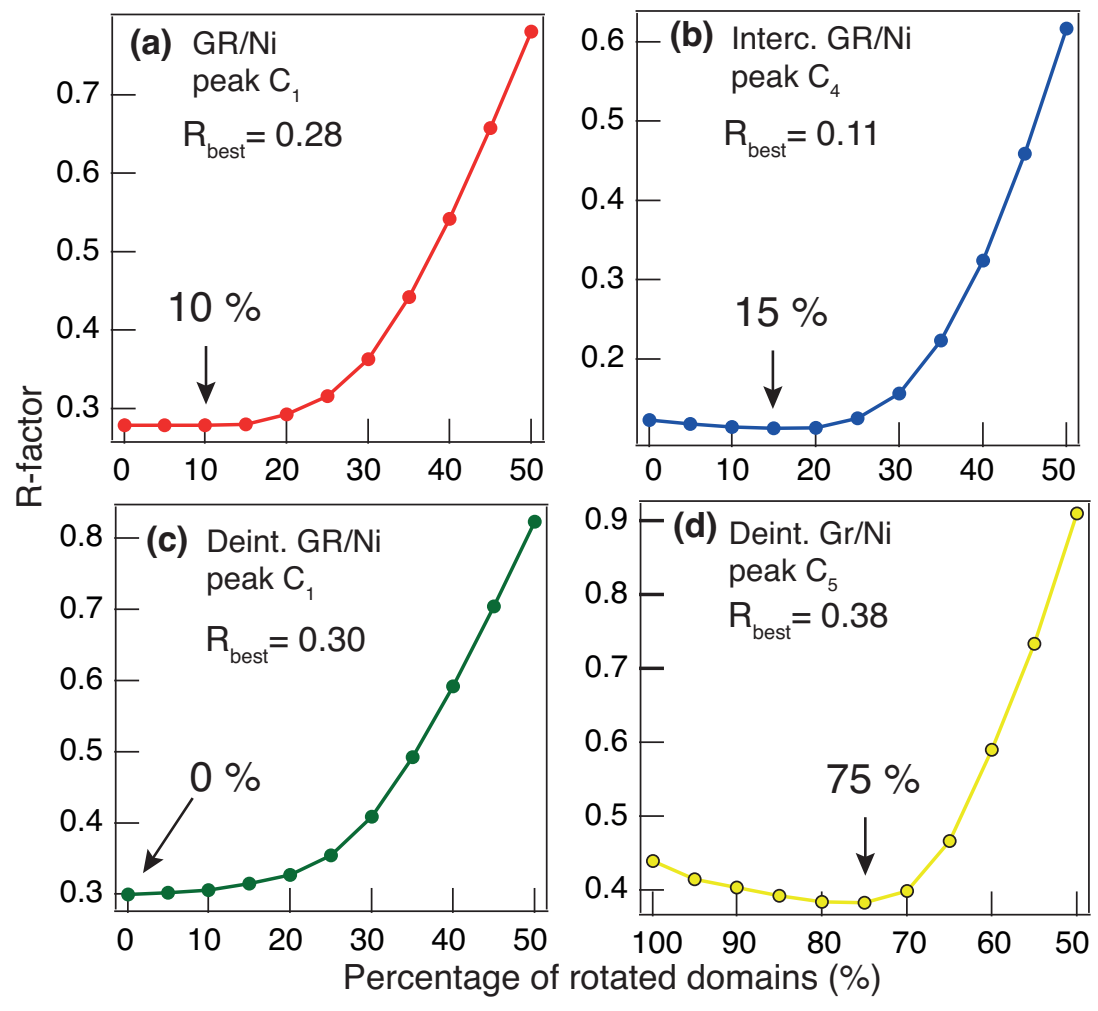

Figure 6. Evolution of the R-factor value for XPD patterns acquired at each intercalation and deintercalation stage. The percentage of rotated domains corresponding to the minimum $\mathrm{R}$-factor is reported.

azimuthal scans shown in Figure 5-(g) indicate that the modulation functions $\chi$ for $C_{5}$ acquired at stage (i) of the intercalation and after the deintercalation are identical. Therefore, graphene lying on chemisorbed oxygen, which arises in the first phase of intercalation and remains after the deintercalation, contains predominantly rotated domains, which then play a pivotal role in triggering the intercalation of oxygen under graphene.

For the pristine graphene on $\mathrm{Ni}$ (Figure 5-a) the best R-factor (0.28) was found when comparing the experiment with a simulation including ca. $10 \%$ of rotated domains. The R-factor was essentially constant (differences smaller than 1\%) when spanning the contribution of the rotated domains between 0 and 20\%, as shown in Figure 6-(a). Hence, the presence of a minor fraction of rotated domain has to be taken into account also for the pristine sample.

Analogously, the minimum R-factor (0.11) was obtained for the pattern of intercalated graphene (Figure 5-b) by including a contribution of rotated domains of $15 \%$, essentially the same as for the pristine sample. The presence of the same amount of rotated domains in the pristine sample and in the oxygen intercalated and deintercalated systems is confirmed also by the behaviour of the features associated to the rotated domains in LEED, which display always the same intensity. This implies that oxygen 
intercalation does not alter the morphology of the graphene layer, lifting both aligned and rotated domains. The smaller R-factor for the GR/O/Ni(111) case can be attributed to the lack of well defined adsorption configuration of graphene on the $\mathrm{NiO}$ as compared to $\mathrm{GR} / \mathrm{Ni}(111)$, where fcc-top or top-bridge adsorption sites are present, as shown by other XPD experiments on the pristine GR/Ni(111) [64]. Therefore, the contributions of the substrate to the C 1s XPD pattern can be overlooked, similarly to GR/Ir(111) [55], thus improving the agreement with the simulations for a free standing GR layer.

The analysis of the pattern stemming from the peak $C_{1}$ for the deintercalated sample shown in Figure 5 -(c) returned a $R$-factor $(R=0.30)$ comparable to what obtained for $\mathrm{GR} / \mathrm{Ni}(111)$, indicating that graphene assumed the same adsorption geometry observed for the pristine sample. Remarkably, the best R-factor was achieved when no contributions from the rotated domains was considered, as shown in Figure 6(c). Hence, after deintercalation, the graphene in contact with the substrate originating $C_{1}$ contains a higher fraction of aligned domains with respect of the pristine $\mathrm{Gr} / \mathrm{Ni}(111)$. This demonstrates that during intercalation the aligned domains are the first to land back on the metal, while the rotated domains remain lying on the chemisorbed oxygen.

From all these observations we can give a comprehensive picture of the intercalation and deintercalation of oxygen at the $\mathrm{GR} / \mathrm{Ni}(111)$ interface. The intercalation begins through defects in graphene lifting first graphene rotated domains, under which oxygen is initially chemisorbed. A larger amount of oxygen promotes the formation of $\mathrm{NiO}$ islands that eventually laterally grow and coalesce to form a complete oxide layer, which lifts both aligned and rotated domains. The deintercalation is triggered by the temperature; the oxide layer undergoes structural modifications and is partially reduced by the thermal annealing, leaving areas with chemisorbed oxygen and areas where the pristine $\mathrm{GR} / \mathrm{Ni}(111)$ configuration is recovered. The aligned domains are the first to land back on the clean metal, while rotated domains remain intercalated and retain chemisorbed oxygen.

\section{Conclusions}

We have shown that intercalation of oxygen under graphene on $\mathrm{Ni}(111)$ initiates from domains that are rotated with respect to the main orientation of the crystal and progresses until an oxide layer is formed, which yields a complete decoupling of the

graphene layer from the metal and restore the Dirac cone at the $\bar{K}$ point of the graphene Brillouin zone. The intercalation is harmless for the graphene, fully lifting the whole layer without etching.

Last, we have observed that the intercalated sample is stable up to ca. $500 \mathrm{~K}$, above which, the deintercalation of oxygen starts. Large regions of graphene land back on the metal, whereas domains where graphene is still intercalated with chemisorbed oxygen and oxide are still present.

XPD patterns supported this interpretation, showing that graphene adsorbs on the intercalated oxide without a well-defined configuration and revealing that both domains 
aligned with the substrate and rotated domains are efficiently intercalated with oxygen. The rotated domains are pivotal to the intercalation process, being the first to be intercalated with chemisorbed oxygen. Moreover, we showed that the aligned graphene domains are the first to be landed back on the clean metal during the deintercalation, while oxygen remains chemisorbed under rotated domains even at $680 \mathrm{~K}$.

These results pave the way to the possibility of realising heterostructures and devices based on this interface, where a self-standing graphene layer is decoupled from the ferromagnetic substrate via an oxide layer. The possibility of injecting spin-polarised electrons from the ferromagnetic substrate into graphene would be instrumental to fully exploit the high carrier mobility and the spin transport properties of graphene.

\section{References}

[1] Batzill M 2012 Surface Science Reports 67 83-115

[2] Colombo L, Wallace R M and Ruoff R S 2013 Proceedings of the IEEE 101 1536-1556

[3] Schwierz F, Pezoldt J and Granzner R 2015 Nanoscale 7 8261-8283

[4] Novoselov K S, Mishchenko A, Carvalho A and Castro Neto A H 2016 Science 353

[5] Voloshina E and Dedkov Y 2012 Physical Chemistry Chemical Physics 1413502

[6] Dedkov Y and Voloshina E 2015 Journal of Physics: Condensed Matter 27303002

[7] Preobrajenski, AB, Ng M L, Vinogradov A S and Mårtensson N 2008 Physical Review B 78073401

[8] Varykhalov A, Sánchez-Barriga J, Shikin A M, Biswas C, Vescovo E, Rybkin A, Marchenko D and Rader O 2008 Phys. Rev. Lett. 101(15) 157601

[9] Grüneis A and Vyalikh D V 2008 Physical Review B 77193401

[10] Voloshina E N, Generalov A, Weser M, Böttcher S, Horn K and Dedkov Y S 2011 New Journal of Physics 13113028

[11] Lizzit S, Larciprete R, Lacovig P, Kostov K L and Menzel D 2013 ACS Nano 7 4359-4366

[12] Lizzit S, Larciprete R, Lacovig P, Dalmiglio M, Orlando F, Baraldi A, Gammelgaard L, Barreto L, Bianchi M, Perkins E and Hofmann P 2012 Nano Letters 12 4503-4507

[13] Park Y S, Park J H, Hwang H N, Laishram T S, Kim K S, Kang M H and Hwang C C 2014 Physical Review X 4 031016-9

[14] Omiciuolo L, Hernández E R, Miniussi E, Orlando F, Lacovig P, Lizzit S, Mentes T O, Locatelli A, Larciprete R, Bianchi M, Ulstrup S, Hofmann P, Alfè D and Baraldi A 2014 Nature Communications 55062

[15] Larciprete R, Lacovig P, Orlando F, Dalmiglio M, Omiciuolo L, Baraldi A and Lizzit S 2015 Nanoscale 7 12650-12658

[16] Forti S, Stöhr A, Zakharov A A, Coletti C, Emtsev K V and Starke U 2016 2D Materials 3035003

[17] Warmuth J, Bruix A, Michiardi M, Hänke T, Bianchi M, Wiebe J, Wiesendanger R, Hammer B, Hofmann P and Khajetoorians A A 2016 Phys. Rev. B 93(16) 165437

[18] Brede J, Sławińska J, Abadia M, Rogero C, Ortega J E, Piquero-Zulaica I, Lobo-Checa J, Arnau A and Cerdá J I 2017 2D Materials 4015016

[19] Larciprete R, Ulstrup S, Lacovig P, Dalmiglio M, Bianchi M, Mazzola F, Hornekær L, Orlando F, Baraldi A, Hofmann P and Lizzit S 2012 ACS Nano 6 9551-9558

[20] Grånäs E, Knudsen J, Schröder U A, Gerber T, Busse C, Arman M A, Schulte K, Andersen J N and Michely T 2012 ACS Nano 6 9951-9963

[21] Ulstrup S, Andersen M, Bianchi M, Barreto L, Hammer B, Hornekær L and Hofmann P $20142 D$ Materials 1025002

[22] Sutter P, Sadowski J T and Sutter E A 2010 Journal of the American Chemical Society 132 8175-8179

[23] Zhang H, Fu Q, Cui Y, Tan D and Bao X 2009 The Journal of Physical Chemistry C 113 8296-8301 
[24] Dong A, Fu Q, Wei M, Liu Y, Ning Y, Yang F, Bluhm H and Bao X 2015 Surface Science 634 $37-43$

[25] Voloshina E, Berdunov N and Dedkov Y 2016 Scientific Reports 620285

[26] Karpan V, Giovannetti G, Khomyakov P, Talanana M, Starikov A, Zwierzycki M, van den Brink J, Brocks G and Kelly P 2007 Physical Review Letters 99176602

[27] Tombros N, Jozsa C, Popinciuc M, Jonkman H T and van Wees B J 2007 Nature 448 571-574

[28] Giovannetti G, Khomyakov P, Brocks G, Karpan V, van den Brink J and Kelly P 2008 Physical Review Letters 101026803

[29] Dedkov Y S and Fonin M 2010 New Journal of Physics 12125004

[30] Generalov A V, Voloshina E N and Dedkov Y S 2013 Applied Surface Science 267 8-11

[31] Dahal A and Batzill M 2014 Nanoscale 62548

[32] Cobas E D, van t Erve O M J, Cheng S F, Culbertson J C, Jernigan G G, Bussman K and Jonker B T 2016 ACS Nano 10 10357-10365

[33] Dedkov Y S, Fonin M, Rüdiger U and Laubschat C 2008 Applied Physics Letters 93022509

[34] Weatherup R S, D'Arsié L, Cabrero-Vilatela A, Caneva S, Blume R, Robertson J, Schloegl R and Hofmann S 2015 Journal of the American Chemical Society 137 14358-14366

[35] Ma L, Zeng X C and Wang J 2015 The Journal of Physical Chemistry Letters 6 4099-4105

[36] Ligato N, Caputi L and Cupolillo A 2016 Carbon $100258-264$

[37] Dedkov Y, Klesse W, Becker A, Späth F, Papp C and Voloshina E 2017 arXiv.org arXiv:1702.02351 (Preprint 1702.02351)

[38] Jacobson P, Stöger B, Garhofer A, Parkinson G S, Schmid M, Caudillo R, Mittendorfer F, Redinger J and Diebold U 2012 ACS Nano 6 3564-3572

[39] Africh C, Cepek C, Patera L L, Zamborlini G, Genoni P, Menteş T O, Sala A, Locatelli A and Comelli G 2016 Scientific Reports 619734

[40] Song J, Bernien M, Wu C B and Kuch W 2016 The Journal of Physical Chemistry C 1201546 1555

[41] Abrami A, Barnaba M, Battistello L, Bianco A, Brena B, Cautero G, Chen Q H, Cocco D, Comelli G, Contrino S, DeBona F, Di Fonzo S, Fava C, Finetti P, Furlan P, Galimberti A, Gambitta A, Giuressi D, Godnig R, Jark W, Lizzit S, Mazzolini F, Melpignano P, Olivi L, Paolucci G, Pugliese R, Qian S N, Rosei R, Sandrin G, Savoia A, Sergo R, Sostero G, Tommasini R, Tudor M, Vivoda D, Wei F Q and Zanini F 1995 Review of Scientific Instruments 66 1618-1620

[42] Petaccia L, Vilmercati P, Gorovikov S, Barnaba M, Bianco A, Cocco D, Masciovecchio C and Goldoni A 2009 Nuclear Instruments and Methods in Physics Research Section A: Accelerators, Spectrometers, Detectors and Associated Equipment 606 780-784

[43] Doniach S and Sunjic M 1970 Journal of Physics C: Solid State Physics 3285

[44] García de Abajo F J, Van Hove M A and Fadley C S 2001 Phys. Rev. B 63(7) 075404

[45] Woodruff D P 2007 Surface Science Reports 62 1-38

[46] Grüneis A, Kummer K and Vyalikh D V 2009 New Journal of Physics 11073050

[47] Kim K S and Winograd N 1974 Surface Science 43 625-643

[48] Holloway P H and Hudson J B 1974 Surface Science 43 141-149

[49] Evans S, Pielasze J and Thomas J M 1976 Surface Science 55 644-662

[50] Norton P R, Tapping R L and Goodale J W 1977 Surface Science 65 13-36

[51] Holloway P H 1981 Journal of Vacuum Science and Technology 18 653-659

[52] Roberts M W and Smart R S C 1984 Journal of the Chemical Society 802957

[53] Tyuliev G T and Kostov K L 1999 Physical Review B 60 2900-2907

[54] Gragnaniello L, Allegretti F, Zhan R R, Vesselli E, Baraldi A, Comelli G, Surnev S and Netzer F P 2013 Surface Science $\mathbf{6 1 1}$ 86-93

[55] Lizzit S, Zampieri G, Petaccia L, Larciprete R, Lacovig P, Rienks E D L, Bihlmayer G, Baraldi A and Hofmann P 2010 Nature Physics 6 345-349

[56] Haberer D, Vyalikh D V, Taioli S, Dora B, Farjam M, Fink J, Marchenko D, Pichler T, Ziegler K, Simonucci S, Dresselhaus M S, Knupfer M, Büchner B and Grüneis A 2010 Nano Letters 10 
3360-3366

[57] Kortan A R and Park R L 1981 Phys. Rev. B 23(12) 6340-6347

[58] Zhang W B and Chen C 2015 Journal of Physics D: Applied Physics 48015308

[59] Gottardi S, Müller K, Bignardi L, Moreno-López J C, Pham T A, Ivashenko O, Yablonskikh M, Barinov A, Björk J, Rudolf P and Stöhr M 2015 Nano Letters 15 917-922

[60] Riedl C, Coletti C, Iwasaki T, Zakharov A A and Starke U 2009 Physical Review Letters 103 246804

[61] Mucha-Kruczyński M, Tsyplyatyev O, Grishin A, McCann E, Fal'ko V I, Bostwick A and Rotenberg E 2008 Phys. Rev. B 77(19) 195403

[62] Schröder U A, Grånäs E, Gerber T, Arman M A, Martínez-Galera A J, Schulte K, Andersen J N, Knudsen J and Michely T 2016 Carbon 96 320-331

[63] Jacobson P, Stöger B, Garhofer A, Parkinson G S, Schmid M, Caudillo R, Mittendorfer F, Redinger J and Diebold U 2011 The Journal of Physical Chemistry Letters 3 136-139

[64] Parreiras D E, Soares E A, Abreu G J P, Bueno T E P, Fernandes W P, de Carvalho V E, Carara S S, Chacham H and Paniago R 2014 Phys. Rev. B 90(15) 155454 